\title{
Disodium Cromoglycate as a Controller for Asthma
}

Bronchial asthma has been thought to be a typical allergic disease, but based on recent findings "allergic inflammation" in the airways is thought to play an important role in the pathogenesis of this disease. The allergic inflammation is acute or chronic resulting from the type-I allergic reaction mediated by IgE antibody or from a similar nonimmunological process (1). Accordingly, the NHLBI/WHO Workshop Report, entitled "Global Initiative for Asthma" (GINA) (2), published in 1995, described the definition as follows: "Asthma is a chronic inflammatory disorder of the airways in which many cells play a role, in particular mast cells, eosinophils and $\mathrm{T}$ lymphocytes. In susceptible individuals this inflammation causes recurrent episodes of wheezing, breathlessness, chest tightness, and cough particularly at night and/or in the early morning. These symptoms are usually associated with widespread but variable airflow limitation that is at least partly reversible either spontaneously or with treatment. The inflammation also causes an associated increase in airway responsiveness to a variety of stimuli".

See also p 976.

As the concept of bronchial asthma has changed to a chronic inflammatory disease of the airways, principles for the treatment of asthma have also changed from conventional therapy with bronchodilators as the first line drugs to placing a greater attention on how to control this form of inflammation. From this viewpoint, a stepwise therapeutic approach to the management of chronic asthma has been introduced both in the GINA and in the "Japanese Guidelines for the Treatment of Allergic Diseases" (3) issued in 1993 and revised in 1995, considering the circumstances in Japan.

Medications for asthma are employed to reverse and prevent symptoms and airflow limitations (airway obstruction) and include bronchodilators, antiallergic compounds, steroids, and so on. In the former GINA guideline (2), these medications are divided into two categories, relievers and controllers. Relievers act quickly to reverse bronchoconstriction and its accompanying acute symptoms and include short-acting inhaled and oral $\beta_{2}$-agonists, short-acting theophylline, systemic corticosteroids and inhaled anticholinergics. Controllers are medications used daily on a long-term basis to achieve and maintain control of persistent asthma. They include anti-inflammatory agents and long-acting bronchodilators, i.e. inhaled and systemic corticosteroids, disodium cromoglycate (DSCG), nedocromil sodium, oral antiallergic compounds, sustained-release theophylline, long-acting inhaled and oral $\beta_{2}$-agonists. Anti-inflammatory agents involving antiallergics are demonstrated to inter- rupt the development of airway inflammation and to have a prophylactic and suppressive effect (1-3). The profile of the anti-inflammatory agent for asthma can be summarized as follows (4):

(1) It must inhibit the activation of cells relevant to inflammatory processes in asthma.

(2) It must inhibit the pathophysiological events in asthma, namely bronchoconstriction, late asthmatic reaction, enhanced bronchial reactivity and airway inflammation.

(3) It must have proven efficacy in the treatment of asthma in long-term studies in different asthmatic populations.

(4) It must have proven safely in long-term use.

DSCG, as aforementioned, can be classified as a controller and has been shown to fulfill these criteria in numerous publications. It should be emphasized here that this compound has an excellent safety profile with no evidence of consistent major adverse events after 25 years of clinical usage on a long-term basis (4). However, as a rule for every drug, DSCG sometimes produces paroxysmal side effects involving skin eruptions, anaphylaxis and asthmatic reactions which are thought to be type I allergic reaction mediated, although DSCG is shown to inhibit the IgE-mediated mediator release from human mast cells in a dose-dependent fashion (5). Such an example case is reported by Katayama and his colleagues (6) in this issue who exhibited a near-death asthmatic reaction upon inhalation of DSCG, as evidenced by positive bronchial DSCG challenge. As the authors described, at least 12 patients who experienced possible DSCG-induced asthmatic attack have been reported in Japan.

In Japan in addition to the use of DSCG as a controller (3), this compound is also often employed as a reliever to reverse bronchoconstriction as a mixture with inhaled $\beta_{2}$-agonist, especially in the pediatric clinic, to obtain good clinical outcome (3, 7). However, one should appreciate again that every drug is a double-edged sword that has favorable and unfavorable effects, and that even DSCG is not exceptional. In any event, this case report (6) warns us that we should pay much more attention to the possible adverse reactions of the drugs. Proper use and careful observation of the patients are also necessary to avoid unexpected outcome.

Takemasa NAKAGAWA, MD Department of Internal Medicine, St. Marianna University Toyoko Hospital, 3-435 Kosugi-cho, Nakahara-ku, Kawasaki 211 


\section{References}

1) Nakagawa T. Treatment of chronic asthma. Asian Med J 39: 260, 1996.

2) NHLBI/WHO Workshop Report. Global Initiative for Asthma. Publication No. 95-3659, National Institutes of Health, Bethesda, 1995.

3) Makino S, ed. Guidelines for the Treatment of Allergic Diseases. Life Science Medica, Tokyo, 1995 (in Japanese).

4) Edwards AM. Sodium cromoglycate (Intal) as an anti-inflammatory agent for the treatment of chronic asthma. Clin Exp Allergy 24: 612, 1994.

5) Church MK, Young KD. The characteristics of inhibition of histamine release from human lung fragments by sodium cromoglycate, salbutamol and chlorpromazine. Br J Pharmacol 78: 671, 1983.

6) Katayama H, Yokoyama A, Fujino S, et al. Near-death asthmatic reaction induced by disodium cromoglycate. Intern Med 35: 976, 1996.

7) Nishikawa K. Effect of the regular use of disodium cromoglycate+ salbutamol inhalation on the patients with intractable bronchial asthma. J Jpn Soc Ped Allergol 2: 60, 1988 (in Japanese). 